\title{
Valoración diagnóstica de la Batería Piaget-Head * (Primera parte)
}

\section{Angel Forner **}

Escuela Universitaria Formación Profesorado EGB. Barcelona.

La revisión de pruebas consideradas clásicas en el proceso diagnóstico depara a menudo sorpresas. La innegable evolución de la psicología en los últimos quince o veinte años cuestiona profundamente los criterios de validez y fiabilidad de pruebas, test y baterías elaboradas anteriormente. A pesar de ello, en la práctica psicológica, seguimos utilizando gran número de instrumentos que aparecieron entre los años cuarenta y cincuenta.

Muchos de ellos, la mayoría, con las oportunas revisiones y puestas al día, proceso necesario, no tan sólo por las modificaciones que la psicología científica impone, sino también por la adecuación a las cambiantes situaciones socioculturales. Ahora bien, existen determinadas pruebas que, probablemente por ser complementarias del proceso evaluador, no han sufrido la oportuna revisión, y éste es el caso que nos ocupa al tratar en un reciente informe la revisión y valoración diagnóstica de la Batería PiagetHead.

La aportación que hemos preparado para Infancia y Aprendizaje Está dividida en dos partes que, por su extensión, obligan a publicarse en números diferentes de esta revista. La primera presenta la discusión crítica sobre la Batería Piaget-Head y, en la segunda, nos remitimos al análisis de orden cualitativo recientemente efectuado y sin duda de mayor interés para la práctica psicológica.

* Se trata de una de las partes de un informe sobre el tema de dominancia lateral y orientación derecha-izquierda realizado bajo el patrocinio del Departamento de Investigación del I.C.E. de la Universidad de Barcelona y supervisada, esta parte, por la doctora María Forns Santacana, del departamento de Psicología Evolutiva de la Facultad de Psicología de la Universidad de Barcelona.

** Dirección del autor: Escuela Universitaria de Formación del Profesorado. Núcleo Universitario de Pedralbes. Universidad de Barcelona. 


\section{INTRODUCCION}

A menudo observamos en los diagnósticos la referencia a resultados de la Batería Piaget-Head o test de Derecha-izquierda y Mano, ojo, oreja, especialmente si se trata de diagnósticos de sujetos jóvenes, entre 6 y 12 años, que presentan bajo rendimiento escolar, dificultades en la adquisición de los aprendizajes, trastornos lecto-escritos o inestabilidad psicomotriz.

La mayoría de las veces, el resultado de la aplicación de la Batería se especifica con una "edad de lateralidad» o un «nivel de conocimiento de derecha-izquierda» correspondiente a una determinada edad que puede o no corresponder con la cronológica o mental. Este resultado se obtiene de la suma de las puntuaciones de cada prueba, según el criterio de cada una, y de trasladarlo a la escala de puntuación global.

El nivel de información que puede aportar el dato «edad» o "nivel de conocimiento derecha-izquierda», nos parece poco significativo y difícil de ser utilizado, y a partir de ahí se suscitó nuestro interés por conocer con detalle la Batería, no sólo en su aplicación sino en su elaboración, realizada originalmente por N. Galifret-Granjon durante los años 1951, 52, 53 y 54 .

Fue repasando la elaboración de la Batería cuando observamos la presencia de criterios de construccrón que no quedaban suficientemente claros. N. Galifret-Granjon la elaboró a partir de la selección de pruebas procedentes de J. Piaget:y $\mathrm{H}$. Head de las que recogió con un criterio reduccionista determinados items de las pruebas originales «Hermanos y hermanas, y derechaizquierda» de J. Piaget, y «Mano, ojo, oreja» de H. Head. No debemos olvidar que el primero utilizaba su test para descubrir la progresión de las estructuras intelectuales, y el segundo para la valoración diagnóstica de la afasia.

También, y considerando que la estandarización que utiliza N. Galifret-Granjon, actualmente vigente, era del año 1954 y a partir de una muestra de sujetos franceses (alumnos de escuelas parisinas entre 6 y 14 años), consideramos la oportunidad de asegurar la fiabilidad de la Batería aplicándola a una muestra de niños de nuestro entorno próximo.

De esta manera nos introdujimos en el tema de la lateralidad, estructuración del espacio y lenguaje. De tal forma que el estudio de estas aptitudes, humanas, aparte de interesarnos profundamente, nos condujo paulatinamente a la idea de que la Batería podía presentar problemas de validez, y sobre todo, que era correcto plantearnos si a partir de su aplicación se podía obtener una valoración diagnóstica más explícita.

Nuestro análisis se amplió al investigar los significados que podían tener los diferentes tipos de error que puede cometer un sujeto en la Batería, estudiándolo en un marco clínico.

Hasta aquí los propósitos. La elaboración del presente informe fue encadenando, como sucede a menudo, situaciones con resultados, suposiciones con afirmaciones o negaciones, y criterios personales con su constatación empírica 0 , por el contrario, sugerencias inmediatamente descartables.

El trabajo no proviene de un interés aislado sobre el tema sino que forma parte de un interés nuestro muy concreto sobre las cuestiones referentes al desarrollo y evolución psicomotrices correlacionadas con el lenguaje y formación de la personalidad.

Las conclusiones a las que llegamos, probablemente, no son espectaculares. Seguramente cualquier 
psicólogo que utilice o haya utilizado esta Batería se habrá aproximado necesariamente a ellos $y$, de todas maneras, damos el esfuerzo por bien empleado, pues pensamos que era oportuno reflexionar y ayudar a hacerlo sobre las posibles implicaciones diagnósticas de los resultados.

\section{JUSTIFICACION DE LA REVISION}

La necesidad y justificación de revisar una escala construida queda fuera de toda duda. Por lo menos hay tres razones concretas que justifican tal necesidad (1). En primer lugar, la revisión permanente de una escala permite su perfeccionamiento a través de utilización y aplicación, pudiendo de esta manera eliminar los posibles errores que intervienen en su elaboración. Segundo, la revisión periódica de una escala obliga también a replantear el uso que de ella se ha hecho, debido a que en algunos casos su aplicación puede forzar excesivamente los objetivos y límites para los que fue creada. Por último, la constante evolución social y cultural hace también muy aconsejable la revisión periódica de la escala, pues podría fácilmente suceder que el material o el contenido o las instrucciones o la forma de presentación quedasen desfasadas y resultasen inapropiadas para la población a la que se dirigía.

Sobre lo expuesto anteriormente y refiriéndonos a nuestro trabajo, las dos primeras razones que se indican (eliminación de errores y la interpretación de objetivos y límites al diagnosticar) son motivos suficientes para justificar la revisión.

En primer lugar, encontramos èrores de elaboración a los que esperamos ofrecer una alternativa de solución; sobre el segundo punto, a la vista de los informes psico- lógicos de niños efectuados por profesionales próximos a todos nosotros, creemos que se ha exagerado la interpretación global de la Batería prescindiendo del análisis interno.

\section{BATERIA PIAGET-HEAD, DE NADINE GALIFRET-GRANJON}

\section{Fundamentos de la prueba}

Se trata de una Batería de test de orientación de derecha-izquierda, que consiste en proponer al sujeto una serie de cuestiones cada vez más complejas para poder asegurar la correcta adquisición de los conceptos derecha-izquierda, presentando cuestiones que obligan, para resolverlas, a analizar posiciones relativas entre objetos y efectuar la transposición necesaria para imitar gestos ejecutados por el examinador delante del sujeto.

El éxito en estas pruebas es un indicador de una buena estructuración de los elementos lateralizados del esquema corporal. La gradación de las dificultades permite marcar etapas genéticas entre 6 y 14 años.

Para aumentar la seguridad en la estimación de los resultados y también para disminuir la influencia del azar, se facilita una tabla general de puntuaciones totales que permiten una gradación de edad en edad.

N. Galifret-Granjon insiste en que no se trata de una interpretación de Piaget ni de Head sino de aprovechar pruebas de estos autores que miden la orientación derecha-izquierda.

Vamos a intentar ofrecer un estracto del original evitando en lo posible añadir comentarios para no distraer la atención del lector.

Al iniciar su exposición introductoria, N. Galifret-Granjon insiste en el interés que para el psicólogo puede tener averiguar la posibilidad del niño de orientarse en el 
espacio. Determinadas actividades (aprendizaje lecto-escritor, organización del espacio gráfico, copia gráfica, orientación sobre planos...), implican organización especial, sobre todo el reconocimiento de las coordenadas «arriba-abajo, y derecha-izquierda».

Durante el desarrollo del niño puede haber dificultades a nivel práxico o a nivel cognitivo que imposibiliten la distinción de «derecha-izquierda» con la seguridad y rapidez necesarias.

N. Galifret-Granjon formula en 1954 la Batería Piaget-Head como resultado del estudio y baremación que efectuó durante los años 1950 a 1954 y que ha estado publicada en diversas ocasiones (2). Analiza el grado de complejidad en el reconocimiento de «derecha-izquierda» y las'edades de superación de estos conceptos teniendo como referente el propio cuerpo, a otra persona y las relaciones espaciales entre objetos.

Retoma y reformula dos pruebas. La primera la extrae de la denominada por Piaget «Prueba de derecha-izquierda» y la segunda de la prueba de Head «Mano, ojo, oreja». Con ellas configura una Batería de seis pruebas, que, aplicadas a niños normales de 6 a 14 años, se caracterizan por el éxito en una determinada prueba - de las seis- según la edad, poniéndose de manifiesto una clara evolución genética.

Obtiene la técnica para el test de reconocimiento de "derecha-izquierda" de Le jugement et le raisonnement chez l'enfant de J. Piaget Haciendo la advertencia de que no se trata de una prueba de orientación espacial, sino de "lógica de relacionesi; con la finalidad de mostrar la «relatividad progresiva de las nociones». Originalmente J. Piaget presenta doce pruebas que componen el test «Hermanos y hermanas, y derecha-izquierda» de las que N. Galifret-Granjon selecciona tres, todas ellas del segundo test de J. Piaget.

Las pruebas procedentes de $\mathrm{H}$. Head (3) se escogen según un criterio, a nuestro entender frágil, pues N. Galifret-Granjon selecciona aquéllas que le parecen más relacionadas con la orientación espacial. Head utilizaba pruebas de ejecución gestual y escrita correspondientes a estímulos visuales o auditivos presentados de forma directa o simbólica. En su estudio sobre los trastornos del lenguaje utilizaba sus pruebas, tanto para localizar la naturaleza del conflicto como para evaluar la recuperación del paciente. Head hacía una serie de consideraciones sobre los principios subyacentes a sus pruebas: respecto a la imitación directa - prueba en la que el sujeto debe reproducir ciertos gestos efectuados por el examinador delante suyo, y consistentes en llevar una mano a un ojo u oreja- aun cuando se trate de una tarea sencilla y que precisa poca formulación simbólica, si se le pide a un sujeto con dificultades de derecha-izquierda la resolución se hace compleja. Cualquier afásico es capaz de ejecutar movimientos simples, aun en el caso de que presenten combinados, ahora bien, si se trata de imitar gestos de las manos derecha-izquierda del examinador situado delante suyo, comete errores con mucha facilidad. Para poder imitar el gesto de quien está delante se precisa una cierta formulación y expresión simbólica (4) que permita comprender y ejecutar «que debe hacerse como un giro» o bien «situarse al revés».

La prueba mano, ojo, oreja revela deficiencias en la mayor parte de las formas afásicas, si bien el diagnóstico de la afasia no puede hacerse tan sólo con este test, excepto en sujetos que presentan una grave deficiencia en la comprensión del sentido simbólico (afasia semántica). 
Recordemos que la hipótesis de $\mathrm{H}$. Head consistía en que la orientación derecha-izquierda podía considerarse como una medida de la capacidad de formulación y expresión simbólica, que se encuentra aíectada en la afasia.

Por otra parte, «ejecutar un comportamiento depende de que la acción, pueda ejecutarse de manera directa o que precise una codificación previa a través de palabras o signos. El test mano, ojo, oreja, que hace intervenir de forma coordinada a nivel espacio-motor actos lateralizados, da diferentes resultados si el sujeto afásico imita gesto por gesto ante un espejo o si, por el contrario, ha de imitar en situación de cara a cara, ya que la reproducción correcta implica la ordenación simétrica de la sucesión de gestos, es decir, la concepción y aplicación de un principio de transposición espacial» (5).

Las dificultades de ejecución por parte del paciente, radican en una deficiente verbalización interior y no en la imposibilidad de ejecutar los actos o movimientos solicitados. «Head no interpretaba la afasia como una deficiencia motriz. La perturbación de la dicción y la perturbación gráfica están determinadas por una alteración de la representación simbólica; es decir, por una lesión que afecta al lenguaje interior. De no ser asi, los pacientes no deberían tener dificultades en el test mano, ojo, oreja, donde fracasan casi siempre» (6).

Debemos recordar que la polémi$\mathrm{ca}$, en aquellos años, sobre si el paragramatismo era una deficiencia de orden sensorio-auditivo (similar a la polémica inicial sobre la dislexia cuando se le daba como causa los déficit visuales) fue superada precisamente por los trabajos de Head al considerar que el lenguaje no estaba definido por funciones sensoriales y motoras sino por un elevado nivel de integración de estas funcio- nes que posibilitaba la actividad simbólica.

La polémica surge con Van Voerkom (7) y otros autores que, retomando algunas de las pruebas de Head, especialmente la de mano, ojo, oreja le dan una nueva interpretación basándose en que lo esencial radica en la "orientación espacial», que se denomina como "dirección» contrastando este criterio con el de «pensamiento simbólico». Con este autor resurge de nuevo la problemática sobre la naturaleza de los trastornos afásicos. Para unos provienen de un trastorno en la construcción espacial y en su análisis (descomposición y recomposición), para otros seguirá tratándose de un trastormo del pensamiento simbólico.

Debemos advertir que la cuestión que acabamos de plantear sigue vigente hoy día, con posiciones a favor y en contra de ambas acepciones sobre la naturaleza de los trastornos afásicos. Nuestra postura respecto a la valoración diagnóstica, y sin ignorar el énfasis que puso Van Voerkom en las dificultades de orientación y dirección, se aproxima, más a los planteamientos básicos de Head, contrariamente a lo que parece deducirse de los de $\mathrm{N}$. Galifret-Granjon, que aún insistiendo en que parece haber cierta solidaridad entre pensamiento verbal y estructuración del espacio, no lo hace con demasiada vehemencia.

En la línea de Van Voerkom aparecen trabajos de R. Mourgues (8) que considera la comprensión del espacio como un punto nuclear del pensamiento conceptual. H. Delacroix (9) asegura que el test mano, ojo, oreja ofrece evidencias de realización mental por referencias espaciales, temporales y lógicas, y aparte de indicar que los pacientes tratados por Head presentaban dificultades perceptivas, manifiesta que en adultos este test presupone muy poco lenguaje interior y, por el con- 
trario, una alta capacidad de manipulación de la orientación espacial.

El punto de partida de nuestra crítica debe entenderse considerando dos hechos:

1. Las pruebas que N. GalifretGranjon recogge del test de J. Piaget, a la fuerza discrepan de los objetivos originales, pues mientras ella las utiliza para medir el conocimiento de derecha-izquierda, Piaget daba por afianzado este conocimiento sobre el propio cuerpo y analizaba la repercusión de aplicarlo sobre el otro y en el espacio.

2. Referente a las pruebas de H. Head. ya hemos mencionado la controversia que apareció sobre su naturaleza. $\mathrm{N}$. Galifret-Granjon parece inclinarse por la consideración de.los detractores de la formulación original. Así, nos encontramos ante la duda de si estas pruebas están midiendo desarrollo del lenguaje o estructuración espacial.

Además, hay que tener en cuenta las variaciones que se introducen en la forma de aplicación y valoración. En las pruebas de Piaget se permite -contrariamente al criterio original- puntuar parcialmente cada prueba. En las de Head, se introduce en dos de ellas una situación de retest en siete de los ocho ítems. $Y$ en ambos casos se reduce la cantidad de pruebas.

Ante las diferentes valoraciones diagnósticas de la Batería PiagetHead, que nos sitúan ante la posibilidad de tratar con mecanismos estructurales de organización espacial - de estructuración del lenguaje (para algunos, como hemos visto, un dilema) y considerando la posibilidad de hallarnos ante la confirmación de una y otra aceptación, incluso de ambas a la vez, nos parece de gran utilidad las propuestas que pueden derivarse de la teoría de «niveles en el procesamiento de la información» $(10,11)$, que nos ofrece explicaciones para las alternativas que hemos encontrado: trastornos de espacialización, trastornos de lenguaje, o ambas situaciones, que naturalmente presuponen trastornos de orden cognitivo.

La fuente de errores que un sujeto puede cometer en la Batería no tienen por qué provenir necesaria ni directamente de una alteración del esquema corporal, o de la orientación espacial, o de la estructuración del lenguaje, sino que también pueden provenir de la forma en que opera con la información, que puede ser errónea debido a que la información a procesar precise de la concurrencia del lenguaje o de conceptos espaciales básicos, o de la. estructuración del esquema corporal, o de todos ellos; y consecuentemente se procesa de forma inadecuada. Dicho de otra manera, la Batería no informa directamente - aparte de casos de alta incapacidad física o intelectual- de trastornos en la orientación o en el lenguaje o en el esquema corporal, sino de la forma operativa (adecuada o errónea) que el sujeto elabora. Pensamos que esta operatividad o conceptualización es susceptible de mejorarse por el aprendizaje intencional de estrategias de elaboración de la.información.

Pongamos un ejemplo: por las causas que sean un sujeto se ha retrasado evolutivamente o ha estructurado deficientemente la espacialización, o lo que es lo mismo, en etapas donde el desarrollo motor se acompaña del descubrimiento de relaciones espaciales, esto no se ha producido. Aplicar a este sujeto una prueba como la que analizamos, para llegar a esta conclusión y situarlo en "un nivel de tal o cual edad» lo vemos desafortunado. El resultado 
de llegar a situar al sujeto en «un nivel de tal o cual edad" significa que se coloca en relación a los sujetos del "nivel edad" por lo que respecta al tratamiento de la información de tipo espacial que recibe. Las consecuencias del diagnóstico deberían referirse a las posibilidades que manifiesta (con intervención del aprendizaje) para modificar -no la relación psicomotricidad/estructuración espacial- sino la forma de procesar u operar con una información de este tipo, susceptible de ser generalizada; y esto obligaría a expresar los resultados de la Batería por referencia positiva o negativa con otras pruebas de capacidad.
No sabemos si nos hallamos muy lejos de los actuales planteamientos de N. Galifret-Granjon, pero refiriéndose a las perspectivas teóricas que han orientado la investigación sobre la percepción, la lengua escrita y la lectura, tanto normal como patológica, decía en el «Coloquio sobre dificultades y fracaso en el aprendizaje de la lengua escrita "que en el campo del lenguaje..." las investigaciones de los últimos quince o veinte años tienen el interés de hallarse sustentadas por diversas teorías o modelos: la teoría de la información, según indican C. Languein, 1968 y F. Richadeau, 1969; y las teorías del juego de F. Bresson, 1963».

\section{BREVE RESUMEN DE LAS PRUEBAS ORIGINALES}

\section{DEL TEST «DERECHA-IZQUIERDA» DE J. PIAGET}

- «Muéstrame tu mano derecha.» "La izquierda.» "Muéstrame tu pierna derecha.» «La izquierda.»

- «Dime cuál es mi mano izquierda.» «La derecha.» «Dime cuál es mi pierna izquierda.» "La derecha.»

- (Los ítems anteriores se aplican en situación de cara a cara. Los siguientes precisan de la disposición de tres objetos - una moneda, una llave, un lápiz, una pulsera y un papel).

- (Por referencia al sujeto colocar sobre la mesa una moneda y a la izquierda un lápiz). “¿El lápiz está a la izquierda o a la derecha?» «¿Y la moneda?»

- (El examinador toma en su mano derecha una moneda y en la muñeca izquierda una pulsera.) «Ves esta moneda, ¿la tengo en mi mano izquierda o derecha?» « ¿Y la pulsera?»

- (El sujeto delante de tres objetos alineados sobre la mesa, un lápiz a la izquierda, una llave en el centro y una moneda a la derecha.)

"¿El lápiz está a la izquierda o a la derecha de la llave?»

«¿ de la moneda?»--

"¿La llave está a la izquierda o a la derecha de la moneda?»

“¿Y del lápiz?»

«¿La moneda está a la izquierda o a la derecha del lápiz?»

"¿Y de la llave?»

- (En la misma situación, se sitúa sobre la mesa una llave a la izquierda, un papel en el centro y un lápiz a la derecha, mostrándolo durante 30', luego se tapan con una carpeta y se anotan los resultados.)

«Presta atención, te enseñaré tres cosas. Fíjate bien, luego me dirás cómo están colocados. ¡Atención...! (ejecución). Muy bien, ahora dime: la llave está a la izquierda o a la derecha del papel.» "Y el lápiz...», etc.

Es interesante señalar que Piaget no consideraba correctas las pruebas si todas las respuestas pardiales no lo eran. También debemos indicar que no habia límite de tiempo a fin de-evitar errores debidos a la 
precipitación o falta de atención. Y, sobre todo, que consideraba nula la prueba en el supuesto de error en los primeros ítems, o sea, que exigía en principio un conocimiento de derecha-izquierda sobre sí.
La adaptación de N. GalifretGranjon recoge tres pruebas, correspondientes al conocimiento de derecha-izquierda sobre sí, sobre el otro y relativa a la posición de los objetos.

\section{DEL TEST «MANO, OJO, OREJA» DE H. HEAD}

El paciente sentado delante del examinador deberá imitar una serie de movimientos consistentes en tocar un ojo u oreja con una u otra mano. Debemos asegurarnos, antes de empezar, que el sujeto conoce la localización de su mano derecha e izquierda, y comprende la naturaleza de las pruebas.

La primera prueba consiste en la imitación de unos gestos que el examinador ejecuta.

En la segunda, ante.un espejo, se solicita al paciente que imite los movimientos reflejados que hace el examinador situado a su lado.

La tercera se realiza dando al sujeto una serie de tarjetas donde figuran esquemáticamente representados unos dibujos de una persona ejecutando movimientos del tipo indicado que el paciente debe reproducir sobre si

La cuarta prueba consiste en cumplir órdenes verbales sobre el mismo tipo de movimientos.

La quinta, ofrece al sujeto órdenes escritas, que debe leer en forma silenciosa, luego en voz alta y finalmente ejecutar los movimientos descritos.

Por último, el paciente debe escribir la descripción de los movimientos que efectúa el examinador.

N. Galifret-Granjon eliminó en la construcción de la Batería aquellas pruebas que le parecían exclusivas del estudio de los afásicos como son las que se refieren a órdenes leídas o escritura de movimientos. De esta manera, conservó tres pruebas, dos de ellas referentes a imitación de gestos - del examinador y de una figura esquemática- y otra de realización de movimientos según órdenes verbales.

\section{PRINCIPALES DIFERENCIAS ENTRE LAS PRUEBAS ORIGINALES Y LAS QUE FIGURAN EN LA BATERIA PIAGET-HEAD}

Respecto a las pruebas procedentes de J. Piaget, ya hemos indicado un criterio inicial que no se respeta, el de asegurarnos que el sujeto conoce "su derecha y su izquierda», al igual que sucede con las pruebas de H. Head.
En general, la Batería insiste en la rapidez de ejecución por parte del sujeto contrariamente a las pruebas originales. En las pruebas de J. Piaget no se exigía mantener los brazos cruzados. Sobre el criterio de puntuación, originalmente sólo se admitía el éxito si se presentaba corrección en todos los ítems de una prueba.

En lo que concierne a las pruebas de.H. Head, sorprende que, caracterizando. a la Batería como un instrumento de medida de la orientación espacial que se halla en la base de la adquisición lecto-escritora, $\mathrm{N}$. Galifret-Granjon elimine precisamente las pruebas donde el sujeto debe leer o escribir. Otra modificación consiste en que en la prueba de tarjetas no se dejan manipular éstas al sujeto, teniendo que modificar su tamaño para mostrarlas con claridad a una cierta distancia. Finalmente, cabe cuestionar la oportunidad de retestar en una misma 
prueba diferentes items, puesto que al tratarse de ocho ítems para cada prueba quedan convertidos en quince al repetir siete de ellos(exceptuando la de tarjetas).

La experiencia demuestra que cuando el sujeto no tiene problemas con este tipo de tarea, responde correctamente a todos los ítems. Por el contrario, cuando tiene dificultades, basta con aplicar ocho ítems para detectarlas. Debemos advertir que en nuestra valoración diagnóstica sobre esta Bateria, se pueden apreciar, a nivel cualitativo, algunas particularidades de escasa significación cuando retestamos en determinados sujetos algunos ítems, sobre todo en estrategias, fatiga, persistencia...

\section{LOS DATOS DEL ESTUDIO}

Los resultados que a continuación se reflejan proceden de nuestro estudio, sobre una muestra de 309 sujetos. Debemos indicar que en la totalidad del estudio se analizaron tres muestras, la primera que acabamos de citar y de la que seguidamente daremos datos, la segunda, una muestra de 1.979 sujetos de edades entre 3 y 10 años, a quienes se aplicaron unas pruebas relacionadas con el tema a fin de concretar etapas evolutivas en el conocimiento de "derecha-izquierda» y una tercera de 30 sujetos de 5 a 20 años de donde parten algunas consideraciones muy puntuales sobre la valoración diagnóstica a nivel cualitativo que presentaremos en la segunda parte.

\section{LA MUESTRA}

Todos los niños escogidos cumplen las siguientes condiciones: siguen la enseñanza en un centro de escolaridad normal, corresponde el nivel que cursan con la edad cronológica correspondiente y no presen- tan de forma manifiesta - según criterio de sus profesores- un retraso que haga pensar en la posible repetición o no superación del curso.

Se procuró disponer, de forma equivalente, de sujetos de ambos sexos. La procedencia escolar (siete escuelas de las zonas de Barcelona, Vallés oriental, Osona, Maresma y Baix Llobregat) era de un 62 por 100 de sujetos asistentes a escuelas privadas y el 38 por 100 restante procedente de escuelas públicas.

El número total de la muestra es de 309 sujetos, 148 niños y 161 niñas de edades comprendidas entre 6 y 14 años.

\section{DISTRIBUCION POR EDADES}

\begin{tabular}{|c|c|c|c|}
\hline Edad & $\mathbf{N}$ & Niños & Niñas \\
\hline \multirow[t]{2}{*}{ 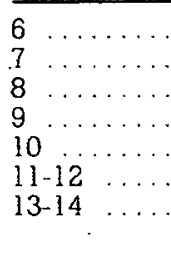 } & $\begin{array}{l}29 \\
32 \\
33 \\
36 \\
40 \\
74 \\
65\end{array}$ & $\begin{array}{l}12 \\
14 \\
16 \\
18 \\
19 \\
37 \\
32\end{array}$ & $\begin{array}{l}17 \\
18 \\
17 \\
18 \\
21 \\
37 \\
22\end{array}$ \\
\hline & 309 & 148 & 161 \\
\hline
\end{tabular}

El criterio de «edad» es el mismo que se propone en la prueba original, produciéndose una pérdida de sujetos de edades inferiores a 11 años al no mantener la diferencia de más/menos dos meses de la edad.

\section{PLANTEAMIENTO DEL PROBLEMA}

Al preparar la redacción del planteamiento del problema para este informe nos vemos obligados a sintetizar los aspectos aproximativos al mismo y exponerlo de forma directa.

En primer lugar, no negaremos, a la vista de los resultados obtenidos, encontrarnos ante un hecho de orden evolutivo, aun cuando como indicaremos en la segunda parte, la ordenación de las diferentes adqui- 
siciones difiere de la presentada por N. Galifret-Granjon.

La principal preocupación radica en analizar si las diferentes pruebas que configuran la Batería miden una misma noción al aumentar progresivamente el índice de dificultad. Nuestra hipótesis experimental manifiesta que la Batería en conjunto, no mide una misma noción sino que son varias las nociones implicadas.

Inmediatamente observamos que las pruebas Head I y $3\left(^{*}\right)$ presentan las mismas características de contenido y, como manífiestan los resultados, las edades de consolidación del concepto empiezan a partir de los 9 años. Por el contrario, la prueba Head 2, sitúa esta edad a partir de 6-7 años, debemos entender que desde la psicología evolutiva no podemos estar midiendo el mismo concepto, o bien puede suceder que desde una perspectiva estructuralista, el concepto medido en Head 2 fuera la génesis de aquel otro que aparece en Head l y 3, cosa que dudamos y remitimos al lector a revisar la última parte de la exposición original de N. GalifretGranjon donde, de forma poco decidida, indica que podríamos hallarnos ante una Batería que mide más de una noción.

Pensamos que, para aclarar estos puntos conflictivos, resulta oportuno el estudio interno que hemos efectuado de la prueba «Derechaizquierda» (Piaget $1,2,3$ ).

Recordemos que las pruebas de «derecha-izquierda» constan, la primera -Piaget l-de dos ítems que solicitan del sujeto la identificación de sus manos derecha y izquierda; la segunda -Piaget 2- propone dos ítems más, que evidencian el conocimiento de la mano derechaizquierda del observador situado delante; y Piaget 3, la tercera, consta de seis ítems que piden las relaciones de situación espacial entre tres objetos alineados ante el sujeto y por referencia propia.
Hay que indicar que en las pruebas Piaget 1 y 2 , al plantear dos preguntas en cada prueba que son de afección binaria - la respuesta sólo puede ser «derecha o izquierda»- el azar puede influir de forma poderosa.

La prueba Piaget 1. («Enséñame tu mano derecha. Tu mano izquierday) sigue el mismo criterio que Piaget daba a su test original no puntuándose como éxito si no se produce en ambos ítems, y arroja los siguientes resultados:

\begin{tabular}{|c|c|c|c|}
\hline Edad & $\mathbf{N}$ & $\begin{array}{c}\mathbf{N} \\
\text { (aciertos } \\
2 \text { items) } \\
\end{array}$ & $\begin{array}{c}\begin{array}{c}\text { Porcentaje } \\
\text { exito }\end{array} \\
\end{array}$ \\
\hline $\begin{array}{l}6 \\
7 \\
7 \\
8 \\
8 \\
9 \\
10\end{array} \ldots \ldots \ldots \ldots \ldots$ & $\begin{array}{l}29 \\
32 \\
33 \\
36 \\
40 \\
74 \\
65\end{array}$ & $\begin{array}{l}27 \\
31 \\
32 \\
36 \\
40 \\
74 \\
65\end{array}$ & $\begin{array}{r}93 \\
97 \\
97 \\
100 \\
100 \\
100 \\
100\end{array}$ \\
\hline
\end{tabular}

Por tanto, y de forma muy similar a los resultados que presenta $\mathrm{N}$. Galifret-Granjon, a los 6 años puede considerarse esta noción como adquirida. Es interesante observar que esta prueba está relacionada con Head 2, que solicita determinadas posiciones o gestos de la mano derecha o izquierda hacia un ojo u oreja, homolateral o no, a partir de órdenes verbales del examinador. En Head 2 encontramos el inicio de resolución entre 6 y 7 años, pudiendo pensarse que se trata del mismo concepto, pero relacionado con dificultades a nivel de «situación, orientación o localización de gestos referidos al propio cuerpon. De alguna manera, esta diferencia (antes de los 6 años en Piaget l, entre 6 y 4 en Head 2). indica el paso entre la adquisición de la noción derechaizquierda y su utilización sobre el propio esquema corporal. Pero si en Head 2 los errores se producen en aquellos ítems donde se solicitan del sujeto actitudes o acciones contralaterales, aquí podemos pensar 
que el sujeto presenta dificultades en el procesamiento de la información o de esquema corporal en cuanto a la sensibilidad propioceptiva, que se caracteriza en las edades inferiores por no resolver correctamente situaciones dicotómicas o con la utilización de dos conceptos antagónicos en una misma operación. Incluso podemos pensar que el error proviene de la situación en que se halle el desarrollo del esquema corporal, sobre todo la imagen de sí mismo. No olvidemos que el estímulo del ítem es de orden oralauditivo y la respuesta de tipo gestual, de tal manera, que el «feedback» autocorrectivo está relacionando la sensibilidad propioceptiva con el lenguaje.

Resumiendo, el nivel de lateralidad que evidencia Head 2 está relacionado con el conocimiento de derecha-izquierda (Piaget 1), pero implica otras nociones procedentes del movimiento, de la sensibilidad propioceptiva y de la imagen de sí mismo como se puede ver fácilmente repasando los resultados de la prueba Head 2 a los 6 años, donde la dispersión típica indica la existencia de errores o rectificaciones del niño, edad a la que ya conóce su derecha-izquierda.

La prueba Piaget 2 ( ¿Cuál es mi mano derecha. Y la izquierda?»), compuesta por dos ítems, y sin admitir margen de error, muestra resultados coincidentes, en general, Con los datos de N. Galifret-Granjon, donde el conocimiento de derecha-izquierda de quien está delante se produce a los 8 años.

\begin{tabular}{|c|c|c|c|}
\hline Edad & $\mathbf{N}$ & $\begin{array}{c}\mathbf{N} \\
\text { (aciertos } \\
\text { 2 fitems) } \\
\end{array}$ & $\begin{array}{c}\text { Porcentaje } \\
\text { éxito }\end{array}$ \\
\hline $\begin{array}{ll}6 & \ldots \\
7 & \ldots \\
8 & \ldots \\
9 & \ldots \\
10 & \ldots \\
11-12 & \\
13-14 & \end{array}$ & $\begin{array}{l}29 \\
32 \\
33 \\
36 \\
40 \\
74 \\
65\end{array}$ & $\begin{array}{l}13 \\
12 \\
20 \\
25 \\
35 \\
70 \\
62\end{array}$ & $\begin{array}{l}44 \\
38 \\
61 \\
69 \\
88 \\
95 \\
95\end{array}$ \\
\hline
\end{tabular}

Consideramos de interés comentar algunas de las situaciones que hemos encontrado al aplicar esta prueba. Caracteriza a los niños menores de 8 años el intento, simulado o no, de girar el tronco para alinearlo en el mismo sentido que el observador, es decir, que no efectúan de forma directa o inmediata la trasposición, sino que precisan de una reconstrucción motórica. Observamos, curiosamente, que en las pruebas de Head 1 y 3 , donde se pide al niño que realice actitudes que se le muestran, no se da el intento de girarse o alinearse en la dirección del modelo. Pensamos que en Head 1 y 3, al no aparecer las palabras «derecha-izquierda» el sujeto obtiene la orientación a partir de conceptos como «girado», «al revés» o «al contrario», como comprobamos en otra de las muestras analizadas en donde se preguntaba a los sujetos qué hacían- para resolver cada una de las pruebas.

Así pues, en la prueba Piaget 2, la operación que hace el niño es para otorgar a una mano u otra el nombre de derecha-izquierda por referencia propia, mientras que en las pruebas Head l y 3 es independiente el nombre (derecha-izquierda) de la acción que se solicita.

Fue a partir de la falta de coincidencia en los niveles-edades para las diferentes pruebas lo que nos aproximaba al planteamiento incial de que la Batería medía, a la vez, diversos conceptos.

Observando la no coincidencia de edades de resolución entre pruebas -que aparentemente miden las mismas nociones- y recapitulando, consideramos: la prueba Piaget 1 manifiesta la adecuación del nombre derecha-izquierda a una parte homolateral del cuerpo. Pensamos que la reducción a dos ítems que hizo $\mathrm{N}$. Galifret-Granjon da mucho margen al azar, que Piaget procuraba reducir al utilizar cuatro ítems. 
La prueba Piaget 2 exige la adecuación de un nombre a la parte 0 lado contralateral de quien está delante en sentido direccional contrario, lo cual obliga a hacer dos operaciones: «transposición» y «nominación». Las pruebas Head 1 y 3 , solicitan "orientación» y reconocimiento de la imagen de si, mientras que Head 2, precisa del conocimiento de la propia derecha-izquierda en una situación de complejidad. Por lo que respecta a la prueba Piaget 3 , por su interés, pasamos a comentarla detalladamente.

Prueba Piaget 3. (Situación relativa de tres objetos.) De entrada se podría pensar que esta prueba, que consiste en dar una orientación lateral a los objetos situados delante del sujeto por referencia a la propia lateralidad, debería ser más fácil de ejecutar que aquéllas que solicitan transposición u orientación espacial, pero esto no sucede así. Posteriormente comentaremos que podría haber un enmascaramiento de la tarea a realizar por parte del sujeto en esta prueba, al estar situada inmediatamente después de la prue- ba Piaget 2, y si bien no podemos dejar de considerarlo, sobre todo en los primeros ítems, creemos que a la vista de los resultados -y, especialmente, considerando una situación experimental que preparamos - además de la posibilidad de enmascaramiento, hay otras circunstancias que dificultan la resolución de la prueba en edades inferiores.

\section{RESULTADOS DE LA PRUEBA PIAGET 3}

\begin{tabular}{|c|c|c|c|}
\hline Edad & $\mathbf{N}$ & $\begin{array}{c}\mathbf{N} \\
\text { (aciertos } \\
\mathbf{6} \text { items) } \\
\end{array}$ & $\begin{array}{c}\text { Porcentaje } \\
\text { éxito }\end{array}$ \\
\hline 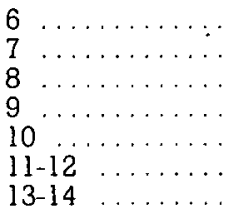 & $\begin{array}{l}29 \\
32 \\
33 \\
36 \\
40 \\
74 \\
65\end{array}$ & $\begin{array}{r}4 \\
4 \\
11 \\
12 \\
16 \\
46 \\
48\end{array}$ & $\begin{array}{l}14 \\
13 \\
33 \\
33 \\
40 \\
62 \\
74\end{array}$ \\
\hline
\end{tabular}

Antes del análisis de los resultados, ofrecemos por su excepcional interés una tabla de datos donde figuran los resultados parciales y acumulados de los sujetos que obtienen 0 ó l error.

\begin{tabular}{|c|c|c|c|c|c|c|}
\hline Edad & $\mathbf{N}$ & $\begin{array}{c}\text { Sujetos } \\
\text { O errores }\end{array}$ & $\begin{array}{c}\text { Sujetos } \\
0-1 \text { errores }\end{array}$ & $\begin{array}{c}\text { Porcentaje } \\
\text { sujetos } \\
\text { O errores }\end{array}$ & $\begin{array}{l}\text { Porcentaje } \\
\text { sujetos } \\
0-1\end{array}$ & $\begin{array}{c}\text { Porcentaje } \\
\text { sujetos } \\
+ \text { de } 1 \\
\end{array}$ \\
\hline 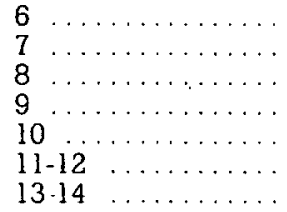 & $\begin{array}{l}29 \\
32 \\
33 \\
36 \\
40 \\
74 \\
65\end{array}$ & $\begin{array}{r}1 \\
3 \\
5 \\
10 \\
19 \\
13\end{array}$ & $\begin{array}{r}5 \\
4 \\
14 \\
17 \\
26 \\
65 \\
61\end{array}$ & $\begin{array}{r}3 \\
9 \\
14 \\
25 \\
26 \\
20\end{array}$ & $\begin{array}{l}17 \\
13 \\
42 \\
47 \\
65 \\
88 \\
94\end{array}$ & $\begin{array}{l}86 \\
87 \\
67 \\
67 \\
60 \\
38 \\
26\end{array}$ \\
\hline
\end{tabular}

A la vista de la primera tabla de resultados de la prueba Piaget 3 , deberíamos esperar a 11-12 años para situarnos en la etapa decisiva con un 62 por 100 de éxitos -coincidiendo con los resultados de $\mathrm{N}$. Galifret-Granjon-. Ahora bien, observando la segunda tabla que presentamos, se ve claramente que con el margen de un error el nivel se sitúa entre 9 y 10 años. Debemos considerar que la prueba consta de seis ítems y que un error, a menudo, se comete por causas ajenas a la prueba: precipitación, ansiedad, etcétera, que consideradas desde el punto de vista cualitativo durante el diagnóstico, debe procurarse aislar de los resultados cuantitativos cuando no influyen en ellos de forma sistemática. 
Datos de los sujetos que en Piaget 3 presentan un error.

\begin{tabular}{|c|c|c|}
\hline Error en el ítem & $\mathbf{N}$ & Porcentaje \\
\hline 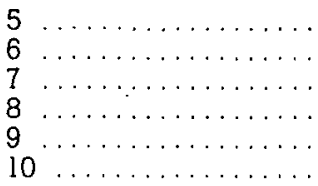 & $\begin{array}{r}6 \\
9 \\
12 \\
9 \\
8 \\
7\end{array}$ & $\begin{array}{l}12 \\
18 \\
23 \\
18 \\
15 \\
14\end{array}$ \\
\hline
\end{tabular}

(La agrupación de los ítems en en la prueba es: 5 y 6,7 y 8,9 y 10 .)

Observamos que el porcentaje más elevado de error aparece en el segundo grupo, ítems 7 y 8 , que piden situar un objeto que está en medio de otros dos, añadiendo una nueva dificultad de estructuración espacial de doble relación antagónica sobre un mismo objeto. El ítem 6 , una vez superado el 5, pide situar un objeto «más alejado» o con otro «entre medias» por referencia al primero.

Como se verá, en la prueba Piaget 3 se ponen de manifiesto diferentes conceptos. En la segunda parte de nuestro estudio, al plantear una prueba donde hemos aislado dos conceptos presentes en Piaget 3 como «distancia» y "relatividad de situación espacial» obtenemos mucho antes la edad de superación al medir solamente el concepto «derecha-izquierda» ante el espacio y los objetos respecto al propio cuerpo.
Recapitulando, creemos que en Piaget 3 puede haber contaminación de la prueba preced mnte -Piaget 2 , que obliga a trasponer- comprometiendo el éxito en los ítems números 5, 6,9 y 10. De tal manera que si el éxito en Piaget l favorecía la superación de.Piaget 2 , el éxito 0 . fracaso en ésta desorienta al niño pequeño en Piaget 3 , ya que ha ejecutado una operación de transposición que ahora no debe realizar en absoluto; y aparece la duda de hasta qué punto estamos valorando intrínsecamente el concepto «derecha-izquierda) o la facilidad en variar esquemas operativos (analizar lo que se pide y ejecutarlo sin que el procesamiento quede contaminado por las pruebas antes efectuadas). De forma similar, en Head I y 3 , la ejecución de la primera en "espejo» o de forma errónea comporta resultados negativos en la 3.

Tampoco podemos olvidar los condicionamientos que se producen a partir de la utilización de materiales y de la posición que ha de mantener el niño - brazos cruzadosque obligan a una relativa «des-subjetivización» comprometiendo el éxito en Piaget 3, aparte de exigir la aplicación en el espacio y sobre objetos de un conocimiento muy relacionado con la sensibilidad propioceptiva. Con esto pretendemos dar a entender las dificultades internas de esta prueba - Piaget 3-y, por tanto, el aumentó de la edad de resolución.

\section{Referencias}

(1) LOPEz FEal, R., y otros: Apuntes de Psicometria. Universidad de Barcelona, 1976.

(2) ZAZZO, R.: Manual para el examen psicológico del niño. Madrid, Fundamentos, 1976.

(3) Hr:AD, H.: Aphasia and Kindred disorders of speech. Londres, Cambridge, 1926.

(4) CAzAYus, P.: La afasia. Barcelona, Herder, $19^{8}$ I. (Pág. 154) da la definición de H. Head sobre «formulación y expresión simbólica», cuando dice que se trata de una "forma de comportamiento en la que todo símbolo verbal o de otro tipo ordena el paso entre la concepción y la ejecución del acto».

(s) CAZAYUS, 1977. Op. cit. (pág. Is s). 
(6) CAZAYUS, 1977. Op. cit. (pág. 196).

(7) VAN VOERKOM: «La signification de certains éléments de l'intelligence dans la genèse des troubles aphasiques." $J$. de Psycbol, $192 \mathrm{I}$.

(8) Mourgurs, R.: «Disordes of symbolic thinking.» J. de Psychol, $192 \mathrm{I}$.

(9) Del.ACRolx, H.: Langage et la pensé. Paris, Alcan, 1930.

(io) CrAik, F. y LOCKHART, R. S.: "Niveles de procesamiento; un marco para la investigación sobre la memoria." Estudios de Psicologia, 1980, 2.

(1 ) Craik, F y LOCKhaRT, R. S.: «Profundidad de procesamiento y retención de las palabras en la memoria." Estudios de Psicologia, 1980, 2.

* En la elaboración del artículo hemos asumido el conocimiento de la prueba por parte del lector por las obvias dificultades que entrañaria su reproducción.

** Puesto que se trata de un artículo dividido en dos entregas, el resumen conjunto se presentará en la segunda parte. 